\title{
XLIX. On some dynamical conditions applicable to Le Sage's theory of gravitation
}

\section{S. Tolver Preston}

To cite this article: S. Tolver Preston (1877) XLIX. On some dynamical conditions applicable to Le Sage's theory of gravitation, Philosophical Magazine Series 5, 4:26, 364-375, DOI: 10.1080/14786447708639355

To link to this article: http://dx.doi.org/10.1080/14786447708639355

曲 Published online: 13 May 2009.

Submit your article to this journal $\sqsubset \pi$

Џ Article views: 5

Q View related articles $\sqsubset$ 


\section{[ 364 ]}

XLIX. On some Dynamical Conditions applicable to Le Sage's Theory of Gravitation. By S. Tolver Preston*.

\section{No II. $\dagger$}

1. TTHE explanation of gravitation has now risen to the rank 1 of one of the foremost problems of modern science; indeed the day for the ascription of occult qualities to matter is now gone, and phenomena, demand an explanation by the reason. The ascription of an occult quality, so far from throwing light upon a phenomenon, only serves to darken it. The effects of gravity (like all other physical effects) being effects of motion, have, like other physical effects, to be explained. A rational explanation has to be given for the motion of approach of two masses. If we were to make an exception of this case, it might be argued that we might make an exception of other cases; and since all physical effects are effects of motion, we should thus in principle bave nothing to explain at all.

2. The absolute necessity for giving an explanation of gravity being admitted, we may inquire what has hitherto been done in this respect. The only theory worthy of serious consideration, or which has stood any test at all, is the theory put forward by Le Sage of Geneva. An immense advance in dynamies has been made since his day. It therefore behoves any one to ask how far the principles put forward by him admit of being improved and modified according to modern advances in dynamies.

3. To any one who has carefully read Le Sage's theory $\ddagger$, it will be evident that the theory consists mainly in a series of postulates or conditions arbitrarily assumed so as to be adapted to produce the results required. Le Sage assumed (1) the movement of streams of particles coming from an indefinite distance in space and converging towards the visible universe. He even calculates (by a given velocity of motion) the distance those particles would require to bave come which produce gravitation, at a remote epoch of 10,000 years (page 22 ). He therefore calls the particles, from the enormous distance he supposes them to come, "ultramundane particles." Next he assumes, quite arbitrarily, that the particles move uniformly or equally in all directions, this being necessary in order that the action

* Communicated by the Author.

† The other part may be found in the 'Philosophical Magazine' for Septenber 1877. Certain points are recapitulated in the present paper for completeness. A paper "On the Mode of the Propagation of Sound" (Phil. Mag. June 18;7) also bears on the subject.

$\ddagger$ The theory is given in a work entitled Deux Traités de Physique Mécanique, by Pierre Prevost. 
of gravity may be equal in all directions. He computes (roughly at about $3,000,000$ ) the number of different directions in which separate streams of particles would require to be moving in order to produce everywhere that sensible uniformity of pressure which is the characteristic of gravity (page 25). It will be noted that these are all assumptions in themselves entirely arbitrary. He next assumes that the mean velocity of the streams of particles is everywhere the same, and the density ererywhere the same.

4. It will be observed that this theory gives no possible idea as to how such a motion of streams of particles among themselves could be kept up, or naturally maintained. Le Sage attempts to evade the difficulty of the particles encountering each other by assuming them to be so small that "not more than one out of every hundred of the particles meets another during several thousands of years." This only removes the difficulty a step further on, without avoiding it. Indeed it may be observed that the theory, in the state in which Le Sage left it, is little more than a series of postulates, some of them almost as unrealizable as gravity itself. This does not detract from a distinct merit in the origination of the theory; for it must be remembered how little dynamical principles were advanced at Le Sage's time, and how few resources he had to draw upon.

5. I have pointed out (Phil. Mag. Sept. 1877) what (whether already observed by others or not) cannot but be regarded as a somewhat startling fact, viz. that no postulates whatever are required for a dynamical theory of gravitation, but that it may be shown that particles of matter in free motion in space must inevitably of themselves arrange their motions so as to produce the effects of gravity-or the special effects of gravity (variation as the square of the distance \&c.) must be produced from pure dynamics in the case of a system of particles in free motion in space, without any necessity for postulates as to the character of the motion at all. This follows from the principles which have been investigated in connexion with the modern kinetic theory of gases, of which Le Sage was ignorant. For it has been demonstrated by Professor Maxwell, in connexion with the kinetic theory of gases, that particles of matter in free collision among each other in space will automatically arrange their motions so as to move uniformly in all directions, i. e. so that an equal number of particles are moving in any two opposite directions (this being the necessary condition for equilibrium of pressure in a gaseous medium*). This cha-

* I may state that $I$ had independently arrived at this same result in $a$ paper "On the Mode of the Propagation of Sound on the Basis of the 
racter of motion, it may be observed, is the first important condition required by Le Sage's theory-which condition therefore follows as a rigid dynamical fact, not as an arbitrary postulate, as he made it. This motion of the particles uniformly or equally towards all directions is not a mere chance fact, but a rigid adjustment, of such a character that when by any artificial means this mode of motion of the particles is disturbed they will automatically, of themselves, return back to this regular form of motion (i. e. so that an equal number of particles are moving in all directions). The other conditions put forward as postulates by Le Sage, viz. that the density of the streams of particles should be the same in all parts, and the mean velocity the same in all parts, are equally necessary results following from the kinetic theory of gases-not, therefore, postulates at all.

6. The only further condition required is that the mean length of path of the particles, before being intercepted by collision with each other, should be great enough to produce the effects of gravity-i.e. so that the particles of the medium may act as streams upon masses immersed in the medium, or may stream past two opposed masses, which by their mutual screening or sheltering action produce the observed effects of gravity. The mean length of path of a particle depends (as is known) upon its size. One size of particle is not à priori more likely than another. By simply, therefore, making the particles small enough, any mean path, however great, may be attained. We thus observe that all the arbitrary postulates of Le Sage's theory, together with all the effects of gravity, naturally and inevitably follow from the simple admission of the existence of matter in space whose normal state is a state of motion, or the existence of a medium in space constituted according to the kinetic theory of gases. In this way the mode in which the motion of the streams of particles through each other is naturally kept up in a state of dynamical equilibrium, is quite easily explained.

7. Here we do not want an indefinite waste of matter, or an indefinite supply of matter from ultramundane space (as Le Sage imagined) to produce gravity, but gravity is produced by matter or a medium which as a whole is stationary, and whose internal motion is kept up and perfectly naturally maintained by the rigid laws of dynamics. The difficulty of the collisions is here completely got over ; for the collisions of the particles among each other, so far (as Le Sage supposed)

Kinetic Theory," published in the 'Philosophical Magazine' for June 1877, without being aware at that time of Professor Maxwell's result. 
from interfering with the uniformity of their motions, is the very condition which corrects and maintains the uniformity of motion in opposition to external disturbing causes. This is the main point which was utterly inexplicable in Le Sage's theory; for it was impossible to see, in the way he put it, how such a motion of streams of particles should be kept up uniformly in all directions, under continual collisions with themselves and with mundane matter. By the application of the principles of the modern kinetic theory of gases to the case, this point is completely solved. As before remarked, it is almost startling that the particular form of motion which the particles themselves automatically keep up should be precisely that one which is required to produce gravity (or an effect varying as the square of the distance \&c.),--also that it should make the density and mean velocity equal in all parts, which is necessary for the effects of gravity*.

8. It is an interesting fact to observe that the distance through which gravity will act will depend on the range through which the streams of particles are comparatively unimpeded, i.e. on the mean length of path of the particles. By making, therefore, the mean length of path of the particles less than the average distance of the stars, it would follow that the stars do not gravitate towards each other, which satisfies the condition for the stability of the universe. It is evident (as has been already pointed out by others) that the assumption of all the bodies of the universe gravitating towards each other is quite inconsistent with stability; and to the truly philosophical mind any theory which rendered sucn an assumption of instability necessary would be in itself improbable. It is only necessary that the mean length of path of the particles be great enough to produce effects of gravity through-

* I have been informed by Professor Tait that a book published in 1818 by Dr. Blair (Regius Professor of Practical Astronomy in the University of Edinburgh) has come under his notice, in which is sketched $x$ theory of gravitation quite similar to that of Le Sage. A later edition of the book, under the title 'Scientific Aphorisms,' was published in 1827. Dr. Blair attempts to account for the motion of the particles being kept up uniformly in all directions by assuming them to rebound from the interior of a hollow spherical surface, which is supposed to bound the visible universe. Of course he, like Le Sage, was ignorant of the remarkable fact of the particles themselves automatically arranging their motions so as to move uniformly in all directions, which has been brought to light in the investigations .connected with the kinetic theory of gases. The spherical surface is of no use to guide the motions of the particles; for they do this themselves. It may be observed that Fatio and Redeker developed a similar theory to that of Le Sage, to a certain extent. When we thus find several minds arriving at the same result, this forms an additional argument for its truth. 
out the greatest range in which we have observed them, which is but an infinitesimal fraction of the distance of the stars *.

9. It may perhaps be well just to sketch here the mode of action of the medium in producing gravity, the manner in which the intensity is made to vary as the square of the distance, \&c. Let $\mathrm{A}$ (in the annexed diagram) represent a molecule or mass; let $\mathrm{C}$ represent the bounding surface of an imaginary hollow sphere described about $\mathrm{A}$. Then, since the particles of the medium are moving uniformly in all directions, a number of them will be passing in all directions through the imaginary spherical surface C. Only those particles which are passing (sensibly)

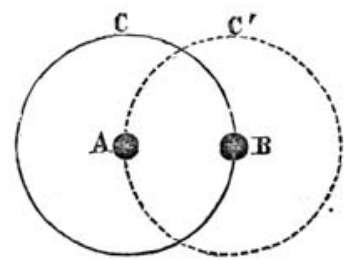
along the radii of the spherical surface will strike $\mathrm{A}$; and therefore we need only regard those special particles which radiate towards $A$. The molecule $A$ being therefore struck equally on all sides, will accordingly remain at rest. But if now we suppose a second molecule to be placed at $B$, then out of the whole number of particles which are directed towards $\mathrm{A}$, the molecule $B$ will intercept a number which is proportional to the area which $B$ cuts off from the whole spherical area $C$. The molecule $A$ will therefore now, owing to the sheltering power of $\mathrm{B}$, be struck with a fewer number of particles in the direction $B A$. The balance of the pressure being thus upset, $A$ will be propelled towards $B$. The same holds true of $B$ relatively to $A$ (on drawing the imaginary spherical surface $\left.\mathrm{C}^{\prime}\right)$. The two molecules $\mathrm{A}$ and $\mathrm{B}$ are therefore propelled towards each other mutually. It now remains to illustrate how the impulsive action varies as the square of the distance. It will be at once evident that since the area of a spherical surface is as the square of the radius, therefore, if $B$

* It may just be noted in connexion with this, that if the above deduction as to the stars not graritating towards each other be true, those stars which have a proper motion must be moving in straight lines.

It is of course evident that all the relations (admirable in their simplicity) existing between the velocity of the particles, the pressure, and density of the medium (or quantity of matter in unit volume), the dimensions, distance, and mean path of the particles, which apply to the kinetic theory of gases, apply equally to the gravific medium,-as also the physical relation existing between the velocity of the particles and the velocity of a wave in a gaseous body, pointed out by me (Phil. Mag. June 1877), the numerical value of which (as determined by Professor Maxwell) was given in the above paper. The assumption is perhaps not unwarranted that it may eventually be possible, by determining the absolute value of some of the above relations, to calculate the mean path, and therefore the range of gravity. 
were removed to a double distance, the imaginary spherical surface described through $B$ with $A$ as a centre, would have four times the area; but the area of the molecule $\mathrm{B}$ remaining constant, B would therefore only shelter $\Lambda$ one fourth the amount it did before; and accordingly $\mathrm{A}$ would be impelled towards B with one fourth the force,-- the same being true of $B$ relatively to $A$.

10. It is found that gravity is proportional to mass. It must therefore be assumed that, owing to the porosity of bodies, or open structure of the molecules, the gravific medium (whose particles are extremely minute) can penetrate freely into the interior of bodies, and thus act upon the internal molecules, so that the total effect is proportional (sensibly) to the number of molecules, or gravity is proportional to mass. Of course this could not hold true with an infinite mass; but it is rigidly demonstrable (by a given degree of porosity) that it could hold true with as near a degree of approximation as experience has shown, and even nearer if necessary. Independent physical reasons for inferring this extreme porosity or permeability of matter will be given further on.

11. My main object in this paper is to meet all possible objections which have been or might be urged against this theory, as, if it be true, it ought to stand against all criticism; and if, on the other hand, it be erroneous, the sooner it is demonstrated so the better. It may just be remarked, in the first place, that in principle there appears to be no other theory conceivable which at all would satisfy the conditions of gravity. Gravity can be referred to two conceivable causes :- (1) to a motion possessed by the molecules of matter themselves, disturbing the equilibrium of pressure of the surrounding medium; (2) to an independent motion of the medium itself acting upon the molecules. The first of these two conditions appears to be imadmissible; for if gravity were due to a motion of the molecules of matter, then since we can readily modify or interfere with the motion of the molecules of matter (as by heat), we could thereby interfere with gravity. The fact, therefore, that it has been found impossible to interfere with gravity, points to a motion in the external medium (which is beyond our control) as the cause. It would be well to keep this fact in view before lightly regarding Le Sage's theory as a mere hypothesis. To the careful observer it will appear to contain rather the essence of a necessary fact, from the absence of (in principle) any other conceivable cause; and if the theory can be shown to be a practical one, consistent with admitted mechanical principles, it will have every condition for acceptance. To us it seems that a closer study of the theory only serves to show its Plil. Mag. S. 5. Vol. 4. No. 26. Nov. 1877. 2 B 
many mechanical beauties and extreme simplicity as a means to an end, satisfactory, not only in the absence of any other conceivable cause, but as affording a perfectly rational conception to the mind of the processes by which the effects are brought about.

12. The most difficult thing perhaps at first sight is to conceive the great permeability or porosity of matter necessary to this theory. It may be perhaps just noted in passing, that most truths are strange at first sight, or else it would be competent for any one to arrive at them. I think it may be shown that this open structure of matter is a thing in itself probable, and also distinctly warranted on independent grounds. In architectural and engineering structure generally we do not observe a solid block formation, if I may so express it, but that open structure which is essential to elasticity and strength. So in molecular architecture, we may not expect to find a mass a solid block, but of open structure, though we naturally cannot see the interstices. Again, the perfectly free passage of light or the waves of xther through a piece of glass, or a wave of obscure heat through a block of rock-salt, also of the magnetic disturbance through all matter, would by itself prove the extremely open structure of natter. There is therefore no difficulty in admitting the openness of structure of matter essential to the dynamical theory of gravitation, as this is in itself a natural thing, pointed to by other facts*.

13. The next difficulty is one pointed out by Professor Maxwell in a notice of Le Sage's theory (Encycl. Brit. 1875, page 46 , under the word "ATOM"). The argument there is that, in view of the demonstrated fact that particles of matter in collision with each other tend to acquire the same kinetic energy, therefore the kinetic energy of a molecule of ordinary matter would ultimately tend to become equal to that of a gravific particle, and that therefore it would appear that the continual impacts of the particles of the gravific medium would necessarily raise matter to an enormous temperature, as the velocity of the gravific particles must of necessity be assumed extremely high. This objection would seem to bave considerable weight; but I think it admits of being surmounted on taking certain facts into consideration. It will be admitted that, in order to produce gravity, it is only neces-

* It may be observed that since, by the rejection of the theory of "action at a distance," all motions developed in matter must inevitably he referred to external media, so therefore, in order that natier may be worked (so to express it) by external media, it must have un open structure-as, in analogy, a steam-engine must have an internal open structure in order that the steam may get at the working parts. 
sary that not less than a certain total of energy should be contained in a given volume of the gravific medium, not that thereby the energy of each particle should necessarily be great. The energy of each particle (whose sum produces a given total of energy) would evidently depend on the number of particles in unit volume. Professor Maxwell assumes that it is "tolerably certain that $\mathrm{N}$, the number of (gravific) corpuscles which are at any one time within unit of volume, is small compared with the value of $\mathrm{N}$ for the molecules of ordinary bodies." Now we may ask, Is this certcin or necessary? for the whole hinges upon this. If, on the contrary, the number of grarific particles in unit volume were not restricted, then by adding to the number of particles, and thus sublividing the total energy among them, the energy of each particle might be made indefinitely small. It might possibly be thought that such a number of particles would be inconsistent with a long free path. But if the subject be considered, it will be observed that a free path of given adequate length may be obtained with an indefinite number of particles, provided the particles be minute-or that, no consequence how numerous the particles (and therefore how small the energy of each), an adequate mean path can be got by reducing their size, their velocity being augmented so as to keep the energy in unit of volume constant. This high velocity of the particles may be shown on other grounds to be a likely condition ; for by this means the whole medium is rendered completely impalpable, or its presence vanishes from the senses-the medium opposing no measurable resistance to the passage of bodies through it. Accordingly, as by a given amount of energy in unit volume the energy of each particle is inversely as their number, so by multiplying the particles the energy of each may be made indefinitely small; and therefore the energy transferred to the molecules of matter would be made indefinitely small, or there would be no measurable rise of temperature at all. This, I submit, removes the difficulty in question.

14. It was pointed out by Le Sage that, in order to explain gravity, it is necessary to assume that the gravific particles rebound from the molecules of matter at a less velocity than they strike. Since, after the average kinetic energy of a molecule of matter has become at least equal to that of a gravific particle, no further transference of energy can take place from the gravific medium to matter (i.e. of course in the case of matter at rest), it is necessary therefore to explain the diminished velocity of rebound of the gravific particles. Sir William Thomson (Phil. Mag. May 1873) has pointed out that this may be a natural consequence of a difference of elastic rigi- 
dity between the gravific particles and the molecules of matter. There may possibly be some who may be inclined to think that this explanation was somewhat forced, or was warranted only as explaining that special case, without being independently likely. I think that, on considering the subject, it will be found that the explanation is in itself highly probable on independent grounds. Where do we find substances in nature whose elastic rigidity is the same. It would be in the highest degree unlikely that portions of matter differing so vastly from each other in dimensions as a molecule and a gravific particle should have the same elastic rigidity. If the elastic rigidity be not the same, it is a strict dynamical fact, not a supposition, that the energy of the particle after its rebound from a molecule, though the same in amount, will not be the same in kind as before; but if the elastic rigidity of the large molecule be greater than that of the minute particle, a part of the translatory motion of the particle will be shivered into vibratory motion at the encounter; and therefore the particle will rebound with a less translatory motion, the deficiency of translatory motion representing the amount converted into vibratory motion at the encounter. It is just as if a tuning-fork, a flexible ring, or any pliable elastic object whatever, were thrown against a hard body (say the hard surface of an anvil), when the body will rebound at a less translatory motion than it struck, the deficiency of translatory motion being compensated for by an accession of vibratory motion. So with a gravific particle striking a molecule of matter; for mere size makes no difference in the principle. It is therefore not an unnatural thing (but highly probable on independent grounds) tha' the gravific particles should have their velocities changed at impact against the molecules of matter. The energy of the particle remains unaltered by the impact; only the distribution of the energy in the particle is changed.

15. The next question is, Do the particles which have thus lost translatory motion and acquired an accession of vibratory motion, recover their normal proportion of translatory motion to viluratory motion again? It has been pointed out by Sir William Thomson that this must be the case. For it bas been demonstrated by Professor Clausius, in connexion with the kinetic theory of gases, that, in the case of a system of particles in free collision among themselves, the relation of the translatory motion to the vibratory motion tends to assume a constant value, so that when this relation is disturbed in any way it is again restored. So, therefore, when the relation of the translatory motion to the vibratory motion of the gravific particles is disturbed by collison with the molecules of matter, this re- 
lation is again restored to its normal value by the collisions of the particles among themselves. This, it may be observed, is therefore a rigid dynamical fact, not an hypothesis.

16. There is therefore no expenditure of energy or work whatever in the maintenance of gravity, since the total amount of energy in the particle is unaltered by collision. Also no supply of energy to the gravific particles is required, since a state of motion is as natural as a state of rest. Furthar, no supply or waste of matter is required for the maintenance of gravity. Le Sage imagined that a continual supply of matter from ultramundane space was necessary. Ho endeavours to gat over this incongruous idea by making the excuse "that nature makes frequently such waste" (page 108). This is evidently no satisfactory excuse at all ; in fact Le Sage, with the limited knowledge of his day, naturally could not get over the difficulty of the collisions, or could not form an idea of the conditions of equilibrium of streams of particles of matter moving in the way he assigned. With our modern knowledge we may deduce that the conditions of equilibrium of such streams of particles are of a perfectly definite character, so as to produce gravity as an inevitable fact. The gravitic medium, therefore, within the bounds of the visible universe is as a whole at rest; and no supply of matter whatever is required. The medium producing gravity is simply a medium constituted as a gas according to the kinetic theory-but quite exceptional in character as regards the extreme minuteness of its particles, their extremely high velocity, and long mean path, the high velocity rendering the medium completely impalpable, or its presence imperceptible to the senses. It is evident that the presence of such a medium could be only rendered directly palpable to the senses by the resistance attendant on the motion of bodies in it. Now it is a known dynamical fact that this resistance diminishes as the velocity of the particles of the medium increases; hence with a given velocity no resistance whatever will be felt, and therefore the presence of the medium must elude detection. These deductions are therefore in perfect harmony with the facts. The mean length of path of the particles of the medium, though great compared with that in the case of ordinary gases, may be considered snall in proportion for a gaseous medium that pervades the area of the visible universe.

17. It would be a wrong idea to imagine that, because the particles of the gravific medium are relatively very close compared with the molecules of ordinary substances, therefore the quantity of matter forming the gravific medium must be relatively great. It is a mathematical fact that the 
total quantity of matter contained in (say a cubic mile) of the medium might be indefinitely small, and yet the particles indefinitely close together, provided the particles are very minute. By a given velocity of the particles of a medium, the unifornity or steadiness of the pressure exerted against matter evidently does not depend on the size of the particles, but on their closeness (which determines the rapidity of succession of the collisions against matter). By a given proximity of particles, therefore, no consequence how minute they may be (and therefore how small the quantity of matter composing the medium), the pressure will remain equally steady. It follows, therefore, that a medium may produce all the uniformity of pressure due to the flow of a continuous fluid, and yet the quantity of matter composing the medium may be indefinitely small. Oring to the frequency of the collisions against matter, due to the close proximity of the particles of the gravific medium and their high velocity, the pressure exerted by the medium is so even and regular as to be imperceptible to the senses, excepting in the effect "gravity." The pressure termed "gravity" due to the motion of the particles of the gravific medium is no more difficult of realization than the pressure of the air due to the motion of its molecules. To get a true idea of the nature of the gravific medium, the conception of extreme closeness of arrangement of the particles, combined with extreme rarity of the medium, must be kept in view. It is easily conceivable, for example, that the particles of a cubic foot of the medium may be in very much closer proximity than the molecules of a cubic foot of lead (from centre to centre), and yet the total quantity of matter contained in a cubic foot of the medium may be less than that contained in a single molecule of lead.

18. The agent producing gravity must therefore not in any way be looked at (as one might possibly be liable to do at first sight) as representing a prodigious quantity of streams of gross matter flying about, but simply as the quiet imperceptible motion of a relatively very small quantity of excessively finely subdivided matter which produces a perfectly uniform pressure, the energy of each particle by itself being totally imperceptible; or only the resultant effect or pressure is noticed, the inexorable motion of the particles (and the resultant effect "gravity") being as incapable of being interfered with as the conservation of energy itself. Surely no more rigid, constant and unalterable cause could be conceived of than that of the normal motion of the particles of a medium among themselves, which, by an inevitable automatic adjustment arrange their motions so as to produce the effects of gravity. "Gra- 
vity" is distinguished by its unalterability under the influence of heat, and general constancy under all conditions. Could a more constant cause be imagined than the above? and could a more simple one be desired? or could any other means of satisfying all the conditions of the problem be conceived of? If simplicity be a mechanical recommendation, the simplicity of the above conditions will recommend themselves. We say simplicity; for surely we have the ne plus ultra of simplicity, when no postulates at all are required, but the total sum of effects may be said simply to evolve themselves out of pure dynamics.

London, October 1877.

L. On Unitation.-VII. Practical Remarks thereon, together with Examples. By W. H. Walenn, Mem. Phys. Soc.*

[Continued from vol, i. p. 549.]

24. TN considering the nature of a unitate and of the ope1 ration of unitation, the method of thought is new to mathematical minds, and does not readily fall in with the routine of ordinary mathematical work; for this reason it is necessary to treat the matter somewhat in detail. The whole subject is fundamental, and is characterized by constant reference to first principles of the simplest kind. It is its very simplicity that creates a difficulty in the facile apprehension of a unitate as a function, and of unitation as an operation. From what has been written it will be perceived, upon examination, that it must by no means be confounded with the remainder to a division*. The denomination of the remainder to a division is determined by the denomination of the last figure of the quotient; but a unitate, in its strictest sense, is an integer ; for it is one of a series of integral values (each member of which is made up of units), the series being determined by the base of the system of unitates, and extending from 1 or unity to the numerical value of the base itself.

Thus, in dividing a given number by 20 , to find the remainder to that division, if the number has a decimal portion, the remainder will be less than unityt. For instance, the division $40 \cdot 123$

$\frac{40 \cdot 123}{20}$ gives for remainder $\cdot 003$; but the unitate of the number $40 \cdot 123$ to the base 20 is 3 , since it must be one of the numbers $1,2,3,4,5,6,7,8,9,10,11,12,13,14,15,16,17$, $18,19,20$. The name unitation is given to the operation to note this distinction in the most marked manner possible. This peculiarity is pointed out in the Philosophical Magazine

* Phil. Mag. [V.] vol. ii. p. 348.

+ Ibid. p. 345 . 